\title{
Counting quadrant walks via Tutte's invariant method
}

\author{
Olivier Bernardi ${ }^{1}$, Mireille Bousquet-Mélou ${ }^{2}$, and Kilian Raschel ${ }^{3}$ \\ ${ }^{1}$ Brandeis University, Department of Mathematics, 415 South Street, Waltham, MA 02453, US \\ ${ }^{2}$ CNRS, LaBRI, Université de Bordeaux, 351 cours de la Libération, 33405 Talence Cedex, France \\ ${ }^{3}$ CNRS, LMPT, Université de Tours, Parc de Grandmont, 37200 Tours, France
}

\begin{abstract}
In the 1970s, Tutte developed a clever algebraic approach, based on certain "invariants", to solve a functional equation that arises in the enumeration of properly colored triangulations. The enumeration of plane lattice walks confined to the first quadrant is governed by similar equations, and has led in the past decade to a rich collection of attractive results dealing with the nature (algebraic, D-finite or not) of the associated generating function, depending on the set of allowed steps.
\end{abstract}

We first adapt Tutte's approach to prove (or reprove) the algebraicity of all quadrant models known or conjectured to be algebraic (with one small exception). This includes Gessel's famous model, and the first proof ever found for one model with weighted steps. To be applicable, the method requires the existence of two rational functions called invariant and decoupling function respectively. When they exist, algebraicity comes out (almost) automatically.

Then, we move to an analytic viewpoint which has already proved very powerful, leading in particular to integral expressions of the generating function in the non-D-finite cases, as well as to proofs of non-D-finiteness. We develop in this context a weaker notion of invariant. Now all quadrant models have invariants, and for those that have in addition a decoupling function, we obtain integral-free expressions of the generating function, and a proof that this series is differentially algebraic (that is, satisfies a non-linear differential equation). This analytic approach solves as well the algebraic model left unsolved in the first part.

Résumé. Nous adaptons à l'énumération des chemins confinés dans le premier quadrant une méthode développée par Tutte dans les années 70 pour compter les triangulations proprement colorées.

Nous prouvons ou reprouvons d'abord ainsi l'algébricité de tous les chemins du quadrant dont la série génératrice est (ou est conjecturée) algébrique ; ceci inclut le célèbre modèle de Gessel. Pour être applicable, la méthode requiert l'existence de deux fonctions rationnelles appelées invariant et fonction de découplage.

Nous passons ensuite à un cadre analytique qui a déjà fourni des expressions intégrales des séries génératrices dans des cas non D-finis, et des preuves de non-D-finitude. Dans ce contexte, nous définissons une notion plus faible d'invariants. Tous les modèles du quadrant admettent alors un invariant, et, pour ceux qui ont de plus une fonction de découplage, nous obtenons une expression sans intégrale de la série génératrice, et prouvons que cette série est différentiellement algébrique (solution d'une équation différentielle polynomiale).

Keywords. Lattice walks, enumeration, differentially algebraic functions, conformal mappings 


\section{Introduction}

In the past decade, the enumeration of plane walks confined to the first quadrant has received a lot of attention, and given rise to many interesting methods and results. Given a set of steps $\mathcal{S} \subset \mathbb{Z}^{2}$ and a starting point (usually $(0,0)$ ), the main question is to determine the generating function

$$
Q(x, y ; t) \equiv Q(x, y)=\sum_{i, j, n \geq 0} q(i, j ; n) x^{i} y^{j} t^{n}
$$

where $q(i, j ; n)$ is the number of $n$-step quadrant walks from $(0,0)$ to $(i, j)$, taking their steps in $\mathcal{S}$. If one only considers walks with small steps (that is, $\mathcal{S} \subset\{-1,0,1\}^{2}$ ), there are 79 inherently different step sets (also called models) and an expression of $Q(x, y)$, sometimes rather complex, is known in each case. Moreover, the nature of this series is also known: it is D-finite (that is, satisfies three linear differential equations, one in $x$, one in $y$, one in $t$, with polynomial coefficients) if and only if a certain group of rational transformations is finite. This happens in 23 cases. In exactly 4 of them, $Q(x, y)$ is even algebraic, that is, satisfies a polynomial equation with polynomial coefficients in $x, y$ and $t$. This classification has been obtained by an attractive combination of approaches: algebraic [BM02, BMM10, Ges86, GZ92], computer-algebraic [BK10, KKZ09, KZ08], analytic [BKR13, Ras12, KR12], asymptotic [DW15, MR09].

The starting point of all of them is a functional equation satisfied by $Q(x, y)$, which is simple to establish, but often hard to solve. For instance, in the case of Kreweras' walks (steps $\nearrow, \leftarrow, \downarrow$ ), it reads

$$
Q(x, y)=1+\operatorname{txy} Q(x, y)+t \frac{Q(x, y)-Q(0, y)}{x}+t \frac{Q(x, y)-Q(x, 0)}{y} .
$$

This is reminiscent of an equation written by Tutte in the 1970 s when studying $q$-colored triangulations:

$$
G(x, y)=x q(q-1) t^{2}+\frac{x y}{q t} G(1, y) G(x, y)-x^{2} y t \frac{G(x, y)-G(1, y)}{x-1}+x \frac{G(x, y)-G(x, 0)}{y} .
$$

Due to the quadratic term, (2) is in fact more complicated than (1). Tutte worked about a decade on this equation, and finally solved it, proving that the series $G(1,0)$ is differentially algebraic, that is, satisfies a (non-linear) differential equation in $t$. One key step in his study was to prove that for certain (infinitely many) values of $q$, the series $G(x, y)$ is algebraic, using a certain notion of invariant [Tut95].

Could this notion bring something new to the classification of quadrant walks? This paper answers this question positively:

- We first adapt Tutte's approach to quadrant walks, and thus obtain short and uniform proofs of algebraicity for all algebraic models (with one small exception). This includes the shortest proof ever found for Gessel's famous model, and extends to models with weighted steps, for which algebraicity was sometimes still conjectural [KY15]. With our approach, a model with finite group is algebraic if and only if it admits a decoupling function (Sections 2 and 3 .

- We then define a weaker notion of invariant, and use it to give an integral free expression of $Q(x, y)$ for models with infinite group that admit a decoupling function (Section 4, see e.g. (14)). We have at the moment found 9 such models. This expression implies that $Q(x, y)$ is differentially algebraic in $x, y$ and $t$ (Section 5). 
We now introduce some basic tools in the study of quadrant walks with small steps. A simple step-bystep construction of these walks gives the following functional equation [BMM10]:

$$
K(x, y) Q(x, y)=K(x, 0) Q(x, 0)+K(0, y) Q(0, y)-K(0,0) Q(0,0)-x y
$$

where

$$
K(x, y)=x y\left(t \sum_{(i, j) \in \mathcal{S}} x^{i} y^{j}-1\right)
$$

is the kernel of the model. It is a polynomial of degree 2 in $x$ and $y$, which we often write as

$$
K(x, y)=\widetilde{a}(y) x^{2}+\widetilde{b}(y) x+\widetilde{c}(y)=a(x) y^{2}+b(x) y+c(x) .
$$

We shall also denote

$$
K(x, 0) Q(x, 0)=R(x) \quad \text { and } \quad K(0, y) Q(0, y)=S(y) .
$$

Note that $K(0,0) Q(0,0)=R(0)=S(0)$, so that the basic functional equation 3 ) reads

$$
K(x, y) Q(x, y)=R(x)+S(y)-R(0)-x y .
$$

Seen as polynomial in $y$, the kernel has two roots $Y_{0}$ and $Y_{1}$, which are Laurent series in $t$ with coefficients in $\mathbb{Q}(x)$. If the series $Q\left(x, Y_{i}\right)$ is well defined, setting $y=Y_{i}$ in (5) shows that

$$
R(x)+S\left(Y_{i}\right)=x Y_{i}+R(0) .
$$

If this holds for $Y_{0}$ and $Y_{1}$, we have

$$
S\left(Y_{0}\right)-x Y_{0}=S\left(Y_{1}\right)-x Y_{1} .
$$

The group of the model, denoted by $G(\mathcal{S})$, is generated by the following two rational transformations:

$$
\Phi(x, y)=\left(\frac{\widetilde{c}(y)}{\widetilde{a}(y)} \frac{1}{x}, y\right) \quad \text { and } \quad \Psi(x, y)=\left(x, \frac{c(x)}{a(x)} \frac{1}{y}\right) .
$$

Both are involutions, thus $G(\mathcal{S})$ is a dihedral group, which, depending on the step set $\mathcal{S}$, is finite or not.

A step set $\mathcal{S}$ is singular if each step $(i, j) \in \mathcal{S}$ satisfies $i+j \geq 0$.

Notation. For a ring $R$, we denote by $R[t]$ (resp. $R[[t]]$ ) the ring of polynomials (resp. formal power series) in $t$ with coefficients in $R$. If $R$ is a field, then $R(t)$ stands for the field of rational functions in $t$. This notation is generalized to several variables. For instance, the series $Q(x, y)$ belongs to $\mathbb{Q}[x, y][t]]$.

\section{A new solution of Gessel's model}

This model, with steps $\rightarrow, \nearrow, \leftarrow, \swarrow$, appears as the most difficult model with a finite group. Around 2000, Ira Gessel conjectured that the number of $2 n$-step quadrant walks starting and ending at $(0,0)$ was

$$
q(0,0 ; 2 n)=16^{n} \frac{(1 / 2)_{n}(5 / 6)_{n}}{(2)_{n}(5 / 3)_{n}}
$$


where $(a)_{n}=a(a+1) \cdots(a+n-1)$ is the rising factorial. This conjecture was proved in 2009 by Kauers, Koutschan and Zeilberger [KKZ09]. A year later, Bostan and Kauers [BK10] proved that the three-variate series $Q(x, y ; t)$ is not only D-finite, but even algebraic. Two other proofs of these results have been given [BKR13, BM15]. Here, we give yet another proof based on Tutte's idea of invariants.

The basic functional equation (5) holds with $K(x, y)=t\left(y+x^{2} y+x^{2} y^{2}+1\right)-x y, R(x)=t Q(x, 0)$, and $S(y)=t(1+y) Q(0, y)$. It follows from $K\left(x, Y_{0}\right)=K\left(x, Y_{1}\right)=0$ that

$$
I\left(Y_{0}\right)=I\left(Y_{1}\right), \quad \text { with } \quad I(y)=\frac{1}{t(1+y)(1+1 / y)}+t(1+y)(1+1 / y) .
$$

We say that $I(y)$ is a (rational) $y$-invariant.

Let us now take $x=t+t^{2}(u+1 / u)$, where $u$ is a new variable. Then it is easy to see that both $Y_{0}$ and $Y_{1}$ are Laurent series in $t$ with coefficients in $\mathbb{Q}(u)$, and that $Q\left(x, Y_{0}\right)$ and $Q\left(x, Y_{1}\right)$ are well defined. Hence (7) holds. Moreover, the kernel equation $K\left(x, Y_{i}\right)=0$ implies that

$$
x\left(Y_{0}-Y_{1}\right)=\frac{1}{t\left(1+Y_{1}\right)}-\frac{1}{t\left(1+Y_{0}\right)},
$$

so that we can rewrite (7) as

$$
J\left(Y_{0}\right)=J\left(Y_{1}\right), \quad \text { with } \quad J(y)=S(y)+\frac{1}{t(1+y)} .
$$

This should be compared to $(8)$. The connection between $I(y)$ and $J(y)$ will stem from the following lemma, the proof of which is elementary.

Lemma 1 Let $F(y)$ be a Laurent series in $t$ with coefficients in $\mathbb{Q}[y]$, of the form

$$
F(y)=\sum_{0 \leq j \leq n+n_{0}} a(j, n) y^{j} t^{2 n}
$$

for some $n_{0} \geq 0$. Then for $x=t+t^{2}(u+1 / u)$, the series $F\left(Y_{0}\right)$ and $F\left(Y_{1}\right)$ are well defined Laurent series in $t$, with coefficients in $\mathbb{Q}(u)$. If they coincide, then $F(y)$ is in fact independent of $y$.

The above series $I$ and $J$ do not satisfy the assumptions of the lemma, as their coefficients are rational in $y$ with poles at $y=0,-1$ (for $I$ ) and $y=-1$ (for $J$ ). Still, we can construct from them a series $F$ satisfying the assumptions of the lemma. First, we eliminate the pole of $I$ at 0 by forming the $y$-invariant $(J(y)-J(0)) I(y)$. The coefficients of this series have a pole of order at most 3 at $y=-1$. By subtracting an appropriate series of the form $a J(y)^{3}+b J(y)^{2}+c J(y)$, we obtain a series satisfying the assumptions of the lemma, which must thus be constant, equal for instance at its value at $y=-1$. In brief,

$$
(J(y)-J(0)) I(y)=a J(y)^{3}+b J(y)^{2}+c J(y)+d
$$

for some series $a, b, c, d$ in $t$. Expanding this identity near $y=-1$ gives:

$$
a=-t, \quad b=2+t S(0), \quad c=-S(0)+2 S^{\prime}(-1)-1 / t,
$$

and

$$
d=-2 S(0) S^{\prime}(-1)-3 S^{\prime}(-1) / t+S^{\prime \prime}(-1) / t .
$$


Replacing in $(10)$ the series $I$ and $J$ by their expressions (in terms of $t, y$ and $S(y)$ ) gives for $S(y)$ a cubic equation, involving three additional unknown series in $t$, namely $S(0), S^{\prime}(-1)$ and $S^{\prime \prime}(-1)$. It is not hard to see that this equation defines a unique power series $S(y)$ in $\mathbb{Q}[y][[t]]$. In the terminology of [BMJ06], this is a cubic equation in one catalytic variable $y$. The solutions of such equations are always algebraic, and a procedure for solving them is given in [BMJ06]. Applying it shows in particular that $S(0) / t, S^{\prime}(-1) / t$ and $S^{\prime \prime}(-1) / t$ belong to $\mathbb{Q}(Z)$, where $Z$ is the unique series in $t$ with constant term 1 satisfying $Z^{2}=1+256 t^{2} Z^{6} /\left(Z^{2}+3\right)^{3}$. Due to lack of space, we do not give any details, but refer the reader to [BM15, Sec. 3.4], where an analogous equation satisfied by $R(x)$ is solved.

\section{Extensions and obstructions}

We now formalize the three main ingredients in the above solution of Gessel's model. The first one is clearly the rational invariant $I(y)$ given by 8 .

Definition 2 A rational function $I(y) \in \mathbb{Q}(t, y) \backslash \mathbb{Q}(t)$ is said to be a $y$-invariant of a quadrant model $\mathcal{S}$ if $I\left(Y_{0}\right)=I\left(Y_{1}\right)$ when $Y_{0}$ and $Y_{1}$ are the roots of the kernel, solved for $y$. We define $x$-invariants similarly.

Note that $I\left(Y_{i}\right)=\left(I\left(Y_{0}\right)+I\left(Y_{1}\right)\right) / 2$ must then be a rational function of $x$, since it is a symmetric function of $Y_{0}$ and $Y_{1}$.

Lemma 3 If a model has a $y$-invariant $I_{2}(y)$, then it admits $I_{1}(x):=I_{2}\left(Y_{0}\right)=I_{2}\left(Y_{1}\right)$ as $x$-invariant. Moreover, $I_{1}\left(X_{0}\right)=I_{1}\left(X_{1}\right)=I_{2}(y)$.

Note that having invariants is just saying that $I_{1}(x)-I_{2}(y)$ vanishes on the curve $K(x, y)=0$, which alludes to Hilbert's Nullstellensatz. In Gessel's case, $I_{2}(y)$ was the function $I(y)$ of $(8)$, and we find $I_{1}(x)=-t / x^{2}+1 / x+2 t+x-t x^{2}$.

Proposition 4 A quadrant model has rational invariants if and only if the associated group is finite.

Proof: A model with an infinite group cannot have rational invariants: the function $I_{1}(x)$ would take the same value for infinitely many values of $x$, which is not possible for a rational function. Conversely, each of the 23 models with a finite group admits rational invariants, listed in Appendix A of [BBMR15].

In the next section, we introduce a weaker notion of (possibly irrational) invariants, which guarantees that any quadrant model now has a (weak) invariant. One key difference with the algebraic setting of the above section is that the new notion is analytic in nature.

The second ingredient of Section 2 was the identity (9), which we formalize as follows.

Definition 5 A quadrant model is decoupled if there exist rational functions $F(x) \in \mathbb{Q}(x, t)$ and $G(y) \in$ $\mathbb{Q}(y, t)$ such that, as soon as $K(x, y)=0$, one has $x y=F(x)+G(y)$.

This is in fact equivalent to the (apparently weaker) identity $x\left(Y_{0}-Y_{1}\right)=G\left(Y_{0}\right)-G\left(Y_{1}\right)$ (which was (9) in Gessel's case), and a possible choice for $F$ is then $F(x)=x Y_{0}-G\left(Y_{0}\right)=x Y_{1}-G\left(Y_{1}\right)$. In Gessel's case, we had $G(y)=-1 /(t(1+y))$, corresponding to $F(x)=1 / t-1 / x$.

So which models are decoupled? Not all, at any rate: for any model that has a vertical symmetry, the series $Y_{i}$ are symmetric in $x$ and $1 / x$, and so any expression of $x$ of the form $\left(G\left(Y_{0}\right)-G\left(Y_{1}\right)\right) /\left(Y_{0}-Y_{1}\right)$ would be at the same time an expression of $1 / x$. At the moment, we have found 13 decoupled models, shown in Tables 1 and 2, 4 with a finite group (and these are, as one can expect from the algebraicity result of Section 2, those with an algebraic generating function), and 9 with an infinite group. 


\begin{tabular}{|c|c|c|c|c|c|c|}
\hline Model & $\longleftarrow \stackrel{\oiiint}{\lessgtr}$ & 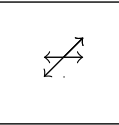 & $\begin{array}{c}1 \underset{\swarrow}{1}{ }^{1} \\
1 \\
\lambda\end{array}$ & 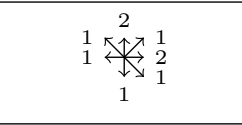 & 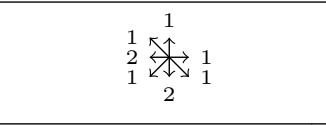 & 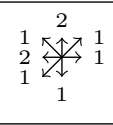 \\
\hline$G$ & $-\frac{1}{y}$ & $-\frac{1}{t(1+y)}$ & $-\frac{1+\lambda t}{t(1+y)}$ & $-y+\frac{1}{y}-\frac{1+3 t}{t(1+y)}$ & $-y^{2}+y\left(1+\frac{1}{t}\right)+\frac{3+\frac{1}{t}}{y}$ & $-y-\frac{1}{y}$ \\
\hline
\end{tabular}

Tab. 1: Decoupling functions for algebraic models (unweighted or weighted). Recall that $F(x)=x Y_{i}-G\left(Y_{i}\right)$.

\begin{tabular}{|c|c|c|c|c|c|c|c|}
\hline Model & $\underset{\# 1}{\stackrel{\bigsqcup}{\longrightarrow}}$ & $\varliminf_{\# 2}^{\uparrow}$ & $\underset{\# 3}{\stackrel{\Psi}{\top}}$ & $\underset{\# 4}{\overleftrightarrow{\leftrightarrows}}$ & $\underset{\# 5}{\sharp}$ & $\underset{\# 6}{\stackrel{1}{\sim}}$ & $\begin{array}{l}\underset{\Downarrow}{\sharp} \\
\# 7\end{array}$ \\
\hline$G$ & $-\frac{1}{y}$ & $-y-\frac{1}{y}$ & $-y-\frac{1}{y}$ & $-y^{2}+\frac{y}{t}+\frac{1}{y}$ & $-\frac{1+t}{t(y+1)}-y$ & $-\frac{1}{y}$ & $-y-\frac{1}{y}$ \\
\hline
\end{tabular}

\begin{tabular}{|c|c|}
\hline$\underset{\# 8}{\stackrel{\uparrow}{\leftrightarrows}}$ & $\underset{\# 9}{\mathbb{\downarrow}}$ \\
\hline$-\frac{1}{y}-y$ & $-\frac{1}{y^{2}}+\frac{1}{t y}+\frac{(t+1) y}{t}-y^{2}$ \\
\hline
\end{tabular}

Tab. 2: Decoupling functions for nine infinite group models.

The final ingredient is the "invariant Lemma" (Lemma 1). It admits analogues for the 4 algebraic models to the left of Table 1, except for the second one. For this model, we were unable to find an analogue of the invariant lemma, and we solve it in a slightly different way (using a weaker form of the invariant lemma) in the next section.

Proposition 6 The existence of rational invariants, decoupling functions and invariant lemma yields new and uniform proofs of the algebraicity of the 4 leftmost models of Table 1, except the second (called reverse Kreweras model). This extends to the 4 weighted models shown on the right of this table.

For Gessel's walks, this is the shortest known proof. For the rightmost weighted model, it is the first one.

Remark. In the finite group case, there exists a systematic procedure to construct the invariants and (when they exist) the decoupling functions, adapting [FIM99, Thm. 4.2.9 and Thm. 4.2.10] to our context. In the infinite group case, the 9 decoupling functions have been guessed-and-checked.

\section{An analytic invariant method}

We now move to an analytic world, and consider $Q(x, y)$ as a function of three complex variables, analytic in the polydisc $\{|x|<1,|y|<1,|t|<1 /|\mathcal{S}|\}$ (at least). This section borrows its notation and several results from the analytic approach of quadrant models [FIM99, Ras12]. The roots $Y_{0,1}$ of the kernel (now called branches of $K$ ) are

$$
Y_{0,1}(x)=\frac{-b(x) \pm \sqrt{b(x)^{2}-4 a(x) c(x)}}{2 a(x)},
$$

where $a, b$ and $c$ are defined by (4). The discriminant $d(x):=b(x)^{2}-4 a(x) c(x)$ has degree three or four, hence there are four branch points $x_{1}, \ldots, x_{4}$ (depending on $t$ ), with $x_{4}=\infty$ if $d(x)$ has degree three. 
We define symmetrically the branches $X_{0,1}(y)$ and their four branch points $y_{\ell}$.

Lemma 7 (|FIM99|) Let $t \in(0,1 /|\mathcal{S}|)$. The $x_{\ell}$ 's are real. Two branch points (say $x_{1}$ and $x_{2}$ ) are in the (open) unit disc, with $\left|x_{1}\right| \leq\left|x_{2}\right|$ and $x_{2}>0$. The other two (say $x_{3}$ and $x_{4}$ ) are outside the (closed) unit disc, with $\left|x_{3}\right| \leq\left|x_{4}\right|$ and $x_{3}>0$. The discriminant $d(x)$ is negative on $\left(x_{1}, x_{2}\right)$ and $\left(x_{3}, x_{4}\right)$, where if $x_{4}<0$, the interval $\left(x_{3}, x_{4}\right)$ stands for $\left(x_{3}, \infty\right) \cup\left(-\infty, x_{4}\right)$.

The branches $Y_{0,1}$ are meromorphic on $\mathbb{C} \backslash\left(\left[x_{1}, x_{2}\right] \cup\left[x_{3}, x_{4}\right]\right)$. On the cuts $\left[x_{1}, x_{2}\right]$ and $\left[x_{3}, x_{4}\right]$, the two branches $Y_{0,1}$ still exist and are complex conjugate. A key object in our definition of weak invariants is the curve $\mathcal{L}$ (depending on $t$ ) defined by

$$
\mathcal{L}=Y_{0}\left(\left[x_{1}, x_{2}\right]\right) \cup Y_{1}\left(\left[x_{1}, x_{2}\right]\right)=\left\{y \in \mathbb{C}: K(x, y)=0 \text { and } x \in\left[x_{1}, x_{2}\right]\right\} .
$$

By construction, it is symmetric with respect to the real axis. If $\mathcal{L}$ is bounded (as for the models of Table 2), we denote by $\mathcal{G}_{\mathcal{L}}$ the domain enclosed by $\mathcal{L}$. Otherwise, $\mathcal{G}_{\mathcal{L}}$ is the domain delimited by $\mathcal{L}$ and containing the real point at $-\infty$. See Figure 1 for examples.
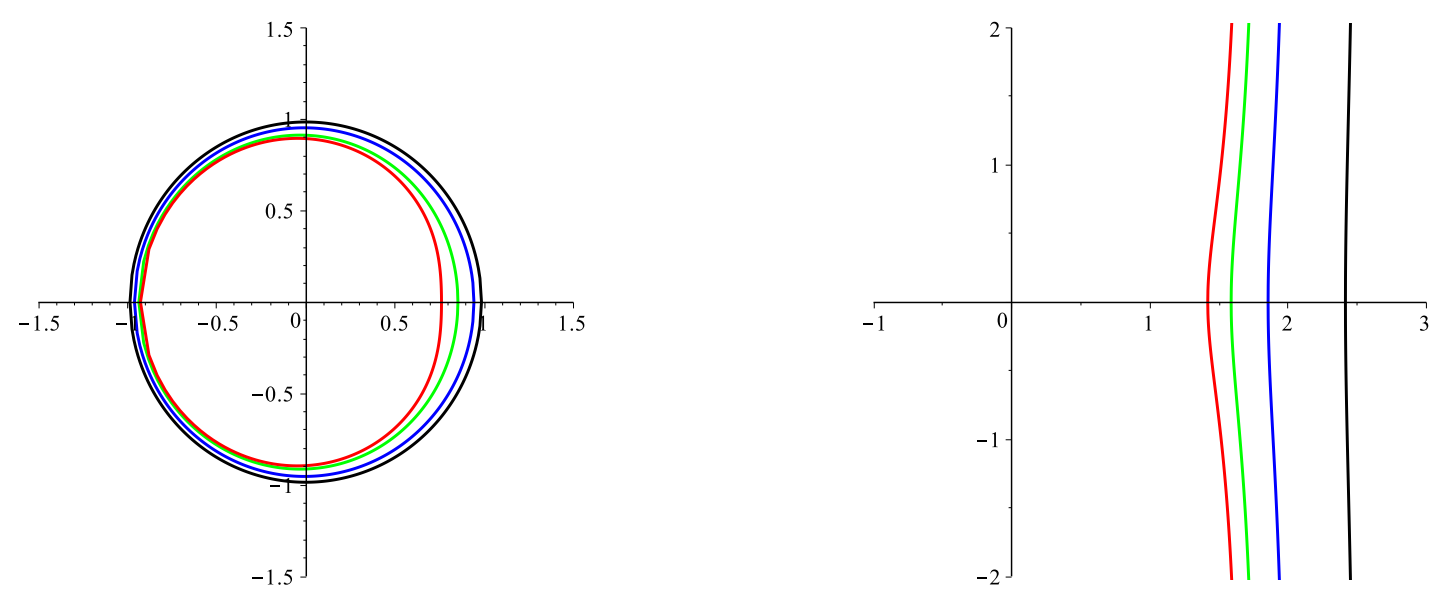

Fig. 1: The curves $\mathcal{L}$ for model \#3 of Table 2 (for $t=0.03,0.1,0.2$ and 0.25 , as one moves closer to the origin) and for the reverse Kreweras model (second model in Table 1 , $t=0.2,0.25,0.28$ and 0.33, from right to left).

The function $S(y)=K(0, y) Q(0, y)$ is analytic in $\mathcal{G}_{\mathcal{L}}$ (by [Ras12, Thm. 5]) and bounded on $\mathcal{G}_{\mathcal{L}} \cup \mathcal{L}$ (this follows from [6] when $x \in\left[x_{1}, x_{2}\right]$ ). Moreover, Eq. (11) of [Ras12] tells us that, for $x \in\left[x_{1}, x_{2}\right]$,

$$
S\left(Y_{0}\right)-x Y_{0}=S\left(Y_{1}\right)-x Y_{1} .
$$

\subsection{Weak invariants}

Definition 8 A function $I(y) \equiv I(y ; t)$ is a weak invariant of a quadrant model $\mathcal{S}$ iffor $t \in(0,1 /|\mathcal{S}|)$ :

- it is meromorphic in the domain $\mathcal{G}_{\mathcal{L}}$, and admits finite limit values on the curve $\mathcal{L}$;

- for any $y \in \mathcal{L}$, we have $I(y)=I(\bar{y})$. 
The second condition is indeed a weak form of the invariant condition $I\left(Y_{0}\right)=I\left(Y_{1}\right)$, because two conjugate points $y$ and $\bar{y}$ of the curve $\mathcal{L}$ are the (complex conjugate) roots of $K(x, y)=0$ for some $x \in\left[x_{1}, x_{2}\right]$. Hence, if the model admits a rational invariant $I(y)$ in the sense of Definition 2 then $I$ is also a weak invariant. However, the above definition is less demanding, and it turns out that every quadrant model admits a (non-trivial) weak invariant, which we now describe (in the non-singular case).

This invariant, traditionally denoted $w(y)$ (or even $\widetilde{w}(y)$ ) in the analytic approach to quadrant problems [FIM99, Ras12], is in addition injective in $\mathcal{G}_{\mathcal{L}}$. In analytic terms, this third condition makes it a conformal gluing function for the domain $\mathcal{G}_{\mathcal{L}}$. Explicit expressions of conformal gluing functions are known in a number of cases (when the domain is an ellipse, a polygon, etc.). In our case the bounding curve $\mathcal{L}$ is a quartic curve [FIM99, Thm. 5.3.3 (i)], and $w$ can be expressed in terms of Weierstrass' elliptic functions (see [FIM99, Sec. 5.5.2.1] or [Ras12, Thm. 6]):

$$
w(y ; t) \equiv w(y)=\wp_{1,3}\left(-\frac{\omega_{1}+\omega_{2}}{2}+\wp_{1,2}^{-1}(f(y))\right),
$$

where the various ingredients of this expression are as follows. First, $f(y)$ is a simple rational function of $y$ whose coefficients are algebraic functions of $t$ :

$$
f(y)= \begin{cases}\frac{\widetilde{d^{\prime \prime}}\left(y_{4}\right)}{6}+\frac{\widetilde{d^{\prime}}\left(y_{4}\right)}{y-y_{4}} & \text { if } y_{4} \neq \infty, \\ \frac{\widetilde{d^{\prime \prime}}(0)}{6}+\frac{\widetilde{d^{\prime \prime \prime}}(0) y}{6} & \text { if } y_{4}=\infty,\end{cases}
$$

where the $y_{\ell}$ 's are the branch points of the functions $X_{0,1}$, and $\widetilde{d}(y)$ is the counterpart of the discriminant $d(x)$ for the variable $y$ (so that $\widetilde{d}\left(y_{4}\right)=0$ ). Then, $\wp_{1,2}$ (resp. $\wp_{1,3}$ ) is Weierstrass' elliptic function with periods $\left(\omega_{1}, \omega_{2}\right)$ (resp. $\left.\left(\omega_{1}, \omega_{3}\right)\right)$, where

$$
\omega_{1}=i \int_{x_{1}}^{x_{2}} \frac{\mathrm{d} x}{\sqrt{-d(x)}}, \quad \omega_{2}=\int_{x_{2}}^{x_{3}} \frac{\mathrm{d} x}{\sqrt{d(x)}}, \quad \omega_{3}=\int_{Y\left(x_{1}\right)}^{x_{1}} \frac{\mathrm{d} x}{\sqrt{d(x)}} .
$$

Note that $\omega_{1} \in i \mathbb{R}_{+}$and $\omega_{2}, \omega_{3} \in \mathbb{R}_{+}$.

It is known that the function $w(y)$ given by (12) is meromorphic in $\mathcal{G}_{\mathcal{L}}$, with a unique pole at $y_{2}$ (which lies indeed in $\mathcal{G}_{\mathcal{L}}$ ). It is an algebraic function of $y$ and $t$ for the 23 models with a finite group, see [Ras12, Thm. 2 and Thm. 3]. It is even rational unless $\mathcal{S}$ is one of the 4 algebraic models. It is then a rational invariant, in the sense of Definition 2 In the infinite group case, $w(y)$ is not algebraic, nor even D-finite w.r.t. to $y$, see [Ras12, Thm. 2]. However, we will prove in Proposition 12 that it is differentially algebraic in $y$ and $t$.

\subsection{The analytic invariant lemma - Application to quadrant walks}

We now come with an analytic counterpart of Lemma 1

Lemma 9 Let $\mathcal{S}$ be a non-singular quadrant model and $I(y)$ a weak invariant for this model. If I has no pole in $\mathcal{G}_{\mathcal{L}}$ (and, in the case of a non-bounded curve $\mathcal{L}$, if I is bounded at $\infty$ ), it is independent of $y$.

This is proved in [Lit00, Ch. 3], in Lemma 1 (resp. 2) for the bounded (resp. unbounded) case. 
Let $\mathcal{S}$ be a quadrant model that is decoupled, in the sense of Definition 5 . In particular,

$$
x\left(Y_{0}-Y_{1}\right)=G\left(Y_{0}\right)-G\left(Y_{1}\right)
$$

for some rational function $G$. Assume that $G$ has no pole on $\mathcal{L}$. Then, by combining (11) with the analytic properties of $S$, we see that

$$
I(y):=S(y)-G(y)
$$

is a weak invariant in the sense of Definition 8 . Since $S$ is analytic in $\mathcal{G}_{\mathcal{L}}$, the poles of $I(y)$ lying in $\mathcal{G}_{\mathcal{L}}$ must be poles of $G(y)$. Let us denote them $p_{1}, \ldots, p_{\ell}$, and assume they are different from the pole $y_{2}$ of $w$. Then there exists a function of the form

$$
r(y)=\sum_{i=1}^{\ell} \sum_{e=1}^{m_{i}} \frac{\alpha_{e, i}}{\left(w(y)-w\left(p_{i}\right)\right)^{e}}
$$

such that $I(y)-r(y)$ has no pole in $\mathcal{G}_{\mathcal{L}}$ and is bounded there - and is still a weak invariant. Applying Lemma 9 tells us that this function is independent of $y$. Let us examine two examples.

Example: Model \#3 of Table 2. This is a decoupled model, with $G(y)=-y-1 / y$. Hence $I(y)=$ $S(y)+y+1 / y=t y Q(0, y)+y+1 / y$ is a weak invariant, with a single pole in $\mathcal{G}_{\mathcal{L}}$, placed at $y=0$ and having residue 1 (the curve $\mathcal{G}_{\mathcal{L}}$ is shown in Figure 1 , left). Thus $I(y)$ differs from $\frac{w^{\prime}(0)}{w(y)-w(0)}$ by a constant, and a series expansion at $y=0$ gives

$$
t Q(0, y)=\frac{1}{y}\left(\frac{w^{\prime \prime}(0)}{2 w^{\prime}(0)}+\frac{w^{\prime}(0)}{w(y)-w(0)}-y-\frac{1}{y}\right)
$$

This argument extends to all models of Table 2 The curve $\mathcal{L}$ is bounded in each case.

Corollary 10 For any of the 9 models of Table 2 the series $Q(0, y)$ admits a rational expression in terms of $y, w(y), w(0), w^{\prime}(0), w^{\prime \prime}(0), w(-1), w^{\prime}(-1), w^{\prime \prime}(-1), w( \pm i), w( \pm j)$, with $j=e^{2 i \pi / 3}$.

Example: the reversed Kreweras model. Recall that this model was left unsolved in Section 3 . The associated curve $\mathcal{L}$ is unbounded (Figure 1, right). This is again a decoupled model, and a decoupling function is $G(y)=-1 / y$ (Table 1 . Accordingly, $I(y)=S(y)+1 / y=t Q(0, y)+1 / y$ is a weak invariant, with a single pole in $\mathcal{G}_{\mathcal{L}}$, placed at $y=0$ and having residue 1 . Further, one can derive from (6) that $I$ is bounded at $\infty$.

Thus $I(y)$ differs from $\frac{w^{\prime}(0)}{w(y)-w(0)}$ by a constant, and a series expansion at $y=0$ gives

$$
t Q(0, y)=-\frac{1}{y}+\frac{w^{\prime}(0)}{w(y)-w(0)}+t Q(0,0)+\frac{w^{\prime \prime}(0)}{2 w^{\prime}(0)} .
$$

It remains to determine $Q(0,0)$. Using the case $i=0$ of 6 and the $x / y$ symmetry, we find that

$$
x Y_{0}=-\frac{1}{x}+\frac{w^{\prime}(0)}{w(x)-w(0)}-\frac{1}{Y_{0}}+\frac{w^{\prime}(0)}{w\left(Y_{0}\right)-w(0)}+t Q(0,0)+\frac{w^{\prime \prime}(0)}{w^{\prime}(0)} .
$$

Specializing for instance at $x=1$ gives an expression of $t Q(0,0)$. In order to prove the algebraicity of $Q(0, y)$ (and hence that of $Q(x, y)$ by (3)), it now suffices to prove that $w(y)$ is algebraic. This can be done by applying Lemma 9 to the rational invariant $I_{2}(y)=t y^{2}-y-t / y$. This gives for $w(y)$ an equation with one catalytic variable, which can be solved in a systematic way using [BMJ06]. 


\section{Differential algebraicity}

As recalled in the introduction, quadrant walks have a D-finite generating function if and only if the associated group is finite - we can now say, if and only if they admit a rational invariant (Proposition 47. Still, one outcome of our analytic invariant approach is that non-linear differential equations hold for a number of models with an infinite group.

Theorem 11 For any of the 9 models of Table 2 the generating function $Q(x, y ; t)$ is differentially algebraic (or D-algebraic) in $x, y, t$. By this, we mean that it satisfies three (non-linear) differential equations with coefficients in $\mathbb{Q}$ : one in $x$, one in $y$ and one in $t$.

The proof builds on Corollary 10 and on the following result, which holds for any non-singular model.

Proposition 12 The conformal gluing function $w(y ; t)$ defined by $(12)$ is D-algebraic in $y$ and $t$.

Proof: (sketched). There are three main steps in the proof:

- We first consider the Weierstrass function $\wp(\omega)$ as a function of $\omega$, but also as a function of its periods $\omega_{1}$ and $\omega_{2}$ or, alternatively, of the values $g_{2}$ and $g_{3}$ (also called invariants in the elliptic terminology!) defined by

$$
g_{k}\left(\omega_{1}, \omega_{2}\right)=\sum_{(i, j) \in \mathbb{Z}^{2} \backslash\{(0,0)\}} \frac{1}{\left(i \omega_{1}+j \omega_{2}\right)^{2 k}} .
$$

Using some differentiation formulæ borrowed from [AS64], we prove that $\wp$ is DA in $\omega, g_{2}$ and $g_{3}$.

- Then, we prove that when $\omega_{1}, \omega_{2}$ are either the functions $\omega_{1}(t), \omega_{2}(t)$ given by (13), or the functions $\omega_{1}(t), \omega_{3}(t)$ (still given by (13)), then the functions $g_{2}$ and $g_{3}$ are DA in $t$. This follows from their expression as modular forms [WW62], and from the fact that modular forms satisfy differential equations [Zag91].

- We conclude using closure properties of D-algebraic functions.

\section{Further results and final comments}

Tutte's invariants offer a new approach of quadrant walks, and we are faced with many open problems, mainly related to the notion of decoupling functions.

- We still need to determine the exact applicability of our approach: which models admit a decoupling function? For a model with finite group, the existence of a decoupling function implies algebraicity. Hence we have found all decoupling functions for walks with finite group. But what about the 51 non-singular models with an infinite group? We have found 9 with a decoupling function and (although not explained in this abstract) we also know that 36 have no decoupling function (this includes for instance the model with jumps $\downarrow, \swarrow, \leftarrow, \nearrow$ ). This leaves 6 more models which might have decoupling functions:

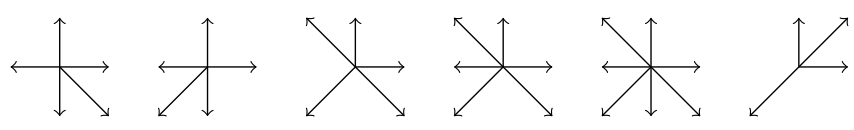


- We have not considered the 5 singular models. But they do admit weak invariants (though not given by (12) and this raises the question of finding decoupling functions for them.

- The known results on the nature of $Q(x, y)$ can be summarized as follows:

\begin{tabular}{|l|c|c|}
\hline & Existence of decoupling functions & No decoupling function \\
\hline Finite group & Algebraic & D-finite transcendental \\
\hline Infinite group & Differentially algebraic & $?$ \\
\hline
\end{tabular}

Could it be that infinite group models without decoupling function have a non-differentially algebraic generating function?

- Can we obtain explicit differential equations in the D-algebraic cases? One possible approach would be to mimic Tutte's solution of (2): he first found a non-linear differential equation valid for infinitely many values of $q$ (for which $G(1,0)$ is in fact algebraic), and then concluded by a continuity argument. In our context, this would mean introducing weights so as to obtain a family of algebraic models converging to a D-algebraic one.

- Another aspect, to be explored in the long version of this extended abstract, is the influence of the starting point on the nature of the generating function. As examples, Gessel's and Kreweras' models starting from $(0,1)$ do not admit decoupling functions, which implies their transcendance.

Acknowledgements. We thank Irina Kurkova for interesting discussions. OB is supported by the NSF grant DMS-1400859. KR is partially supported by the project MADACA from the Région Centre and by the ANR Graal Grant (ANR-14-CE25-0014). We are grateful to one of our referees who spotted mistakes in our tables.

\section{References}

[AS64] M. Abramowitz and I. Stegun. Handbook of mathematical functions with formulas, graphs, and mathematical tables, volume 55 of National Bureau of Standards Applied Mathematics Series. U.S. Government Printing Office, Washington, D.C., 1964.

[BBMR15] O. Bernardi, M. Bousquet-Mélou, and K. Raschel. Counting quadrant walks via Tutte's invariant method (extended abstract). Arxiv:1511.04298, 2015.

[BK10] A. Bostan and M. Kauers. The complete generating function for Gessel walks is algebraic. Proc. Amer. Math. Soc., 138(9):3063-3078, 2010. ArXiv:0909.1965

[BKR13] A. Bostan, I. Kurkova, and K. Raschel. A human proof of Gessel's lattice path conjecture. Trans. Amer. Math. Soc. (to appear), 2013. ArXiv:1309.1023.

[BM02] M. Bousquet-Mélou. Counting walks in the quarter plane. In Mathematics and computer science 2, (Versailles, 2002), Trends Math., pages 49-67. Birkhäuser, Basel, 2002.

[BM15] M. Bousquet-Mélou. An elementary solution of Gessel's walks in the quadrant. ArXiv:1503.08573, 2015. 
[BMJ06] M. Bousquet-Mélou and A. Jehanne. Polynomial equations with one catalytic variable, algebraic series and map enumeration. J. Combin. Theory Ser. B, 96:623-672, 2006. Arxiv:math/0504018.

[BMM10] M. Bousquet-Mélou and M. Mishna. Walks with small steps in the quarter plane. In Algorithmic probability and combinatorics, volume 520 of Contemp. Math., pages 1-39. Amer. Math. Soc., Providence, RI, 2010. ArXiv:0810.4387

[DW15] D. Denisov and V. Wachtel. Random walks in cones. Ann. Probab., 43(3):992-1044, 2015. Arxiv:1110.1254.

[FIM99] G. Fayolle, R. Iasnogorodski, and V. Malyshev. Random walks in the quarter-plane, volume 40 of Applications of Mathematics (New York). Springer-Verlag, Berlin, 1999.

[Ges86] I. M. Gessel. A probabilistic method for lattice path enumeration. J. Statist. Plann. Inference, 14(1):49-58, 1986.

[GZ92] I. M. Gessel and D. Zeilberger. Random walk in a Weyl chamber. Proc. Amer. Math. Soc., 115(1):27-31, 1992.

[KKZ09] M. Kauers, C. Koutschan, and D. Zeilberger. Proof of Ira Gessel's lattice path conjecture. Proc. Nat. Acad. Sci. USA, 106(28):11502-11505, 2009. ArXiv:0806.4300.

[KR12] I. Kurkova and K. Raschel. On the functions counting walks with small steps in the quarter plane. Publ. Math. Inst. Hautes Études Sci., 116:69-114, 2012. ArXiv:1107.2340.

[KY15] M. Kauers and R. Yatchak. Walks in the quarter plane with multiple steps. In FPSAC 2015, DMTCS Proceedings, pages 25-36, 2015. http://fpsac2015.sciencesconf.org/70160.

[KZ08] M. Kauers and D. Zeilberger. The quasi-holonomic Ansatz and restricted lattice walks. J. Difference Equ. Appl., 14:1119-1126, 2008. ArXiv:0806.4318.

[Lit00] G. Litvinchuk. Solvability theory of boundary value problems and singular integral equations with shift. Kluwer Academic Publishers, Dordrecht, 2000.

[MR09] M. Mishna and A. Rechnitzer. Two non-holonomic lattice walks in the quarter plane. Theoret. Comput. Sci., 410(38-40):3616-3630, 2009. ArXiv:math/0701800.

[Ras12] K. Raschel. Counting walks in a quadrant: a unified approach via boundary value problems. J. Eur. Math. Soc. (JEMS), 14(3):749-777, 2012. ArXiv:1003.1362.

[Tut95] W. T. Tutte. Chromatic sums revisited. Aequationes Math., 50(1-2):95-134, 1995.

[WW62] E. T. Whittaker and G. N. Watson. A course of modern analysis. Fourth edition. Cambridge University Press, New York, 1962.

[Zag91] D. Zagier. Modular forms of one variable. Notes based on a course given in Utrecht, Spring 1991. Available at this URL, 1991. 